\title{
Strengthening Maintenance Quality Control of Components to Improve the Reliability of B737NG Airplane Pneumatic System
}

\author{
Zhongjun Chen ${ }^{1, a}$ and Tao Wang ${ }^{2, b}$ \\ ${ }^{1}$ Guangzhou Civil Aviation College, Guangzhou 510403, China \\ ${ }^{2}$ China Southern Airlines Hubei aircraft maintenance Factory, Wuhang 430000,China \\ aczj777380@163.com, bwangtao_a@csair.com
}

\section{Keywords: Pneumatic system; Maintain; Reliability; Monitoring}

Abstract. Through the statistics of the replacement parts of the B737NG aircraft pneumatic system, it is found that the precooler control valve $(\mathrm{PCCV})$ is an important factor leading to the pneumatic system failure. It is concluded that the working conditions and the maintenance quality of the PCCV are the main reasons for the high failure. It is suggested that strengthening the quality management of the parts for repair and monitoring the performance of the components are effective ways to improve the reliability of the aircraft pneumatic system.

\section{Introduction}

The pneumatic system of an aircraft is complex and the system failure can create massive losses ${ }^{[1]}$. Improving reliability of the pneumatic system has become a difficult problem for many years. Airlines have adopted a variety of measures, including promoting pneumatic system health checks, to improve the reliability of aircraft pneumatic system ${ }^{[2]}$. However, the trouble of B737NG pneumatic system still puzzles the airlines. The preventive maintenance of the B737NG pneumatic system has been adopted, but the process causes a large number of accessories to be replaced and maintained in advance, resulting in a sharp rise in cost.

This paper analyzes data from 158 B737NG aircrafts, to find out the parts with the highest failure rate in the aircraft pneumatic system, and discuss the reasons for the high failure rate. Corresponding measures are taken to improve the reliability of the air pneumatic system of the B 737NG aircraft.

\section{Statistical analysis on the replacement parts of the pneumatic system}

Data has been collected from January, 2010 to December, 2011 on 158 B737NG aircrafts. The data was focused on the causes of the pneumatic system malfunction. Each malfunction incident was counted and a total of 757 parts were replaced because of the failure. A total of 12 categories of component replacements were recorded, as shown in Table 1.The replacements of 203 PCCV and 168 high stage regulator, account for approximately $50 \%$ of the total.

Table 1. Statistics of the replacement of pneumatic system parts

\begin{tabular}{|l|l|l|l|l|l|l|l|l|}
\hline Parts & PCCV & HSR & 390F S & BAR & PRSOV & HSV & 450F S & others \\
\hline Times & 203 & 168 & 67 & 70 & 93 & 81 & 45 & 30 \\
\hline Percent & 26.82 & 22.2 & 8.9 & 9.2 & 12.3 & 10.7 & 5.9 & 4.0 \\
\hline
\end{tabular}

According to the analysis of the working principle of the system, the failure of the high pressure stage shutoff valve is mainly related to the low pneumatic pressure of the engine at low power ${ }^{[3]}$, and has little effect on the failure of the engine at high power in the air. So the failure of PCCV is the main cause of pneumatic system malfunction. By analyzing the serial number of the replacement parts, 18 PCCV were repeatedly repaired because of failures, and the replacement and repair frequency were more than 3 times in two years. 


\section{Main reasons for high failure rate of $\mathrm{PCCV}$}

PCCV working condition. The PCCV is a pneumatic control mechanical component, the opening adjustment is accomplished automatically by the servo pressure. Its main function is to change the valve opening according to the engine power to adjust the cooling air quantity through the precooler and reduce engine pneumatic pipe temperature to avoid overheating. The changes of the engine working state and external conditions result in constant fine adjustment of the precooler valve and frequent actuation causes wear and tear of valve parts.

The undesirable repair quality of certain components .Component repair is divided into two cases: failure repair and claim repair. When failed components are sent to repair, it is found that worn and teared parts are replaced in time, and the quality of repair is guaranteed because new parts have taken their place and cost is not considered. When making a claim for repair, the repair factory takes cost into account. The factory often only tests and repairs the parts repaired previously, and many parts were found to have worn out and failed to replace the new parts in time. In some cases, it is simply washed and tagged and returned directly to the airline. Because the repair factory does not investigate the cause of the failure thoroughly, the same component can break down repeatedly, and the reliability of the pneumatic system is reduced.

Measures taken to improve. To ensure the quality of component repairs, the following measures have been taken:

1).Strengthen the repair management of claim parts, the PCCV that failed shortly after a repair should not simply be repaired again, instead, the quality of the accessory repair factory need to be monitored. A good balance between cost and quality need to be maintained.

2).Collect and arrange a list of parts of the control valve, and pay close attention when the valve is repaired.

3).The consistency of satisfactory work of each repair factory is different, resulting in some parts with undesirable quality. The factories with poor repair quality should be eliminated in order to improve the repair quality of the parts.

4).The three key components of the B737NG aircraft pneumatic system with the highest replacement frequency are: the PCCV, the high stage regulator, and the bleed air regulator on the wing. If the service time of a component is consistently lower than average after repair, inspect the manufacturer and the process of parts repair and replacement to determine whether the failure of the component is related to the repair process or to the manufacturer. Avoid repeated failure of the same component to increase the reliability of the pneumatic system.For the same component, if it has failed and repaired three consecutive times in a short period of 1000 flight hours, it will be the focus of attention. First, check the history of maintenance records, if it is due to the repeated failure caused by the claim for repair, send the material to a reliable repair factory for in depth inspection and maintenance; If the component is not a claim and the failure occurs again and again after repair by different repair factories, it shall not be installed.

\section{Strengthening the performance monitoring of the components to improve the reliability of the system}

The most important step to improve the repair quality of the components is to start with the performance monitoring of the components. Take measures to strengthen the real-time monitoring of the components of the pneumatic system in order to find out the hidden troubles in time. Take preventive maintenance measures to replace the relevant system parts in advance of the failure.

Establish a test database of the pneumatic system components.To understand the trend of the performance of each part in the pneumatic system, a test database of the system parts should be set up, and the test data be recorded when the B737NG fleet carries out the pneumatic system health inspection. 
The establishment the test database, enables the staff to understand potential causes of the failure of the pneumatic system components when faced with an engine system malfunction. This way, repair staff can save time and reduce errors when troubleshooting.

Establish a database of critical component usage time. It is key to establish a database of critical component usage time, as shown in Table 2 (TSN :service time of new parts TSR: service time after repair). In wing time, statistics of the three key components of the B737NG aircraft pneumatic system with the highest replacement frequency, the average service life of each component is calculated, and the installation time of the existing engine pneumatic system is compared with the average life data of the component, so as to strengthen the monitoring and control.

Table 2 .PCCV usage time

\begin{tabular}{|c|c|c|c|c|}
\hline PN & SN & TSN & TSR & original \\
\hline $3289562-5$ & 10215 & 12289 & 1332 & T \\
\hline $3289562-5$ & 10059 & 9756 & 226 & F \\
\hline $3289562-5$ & 10718 & 3073 & 575 & F \\
\hline $3289562-5$ & $2586 \mathrm{C}$ & 9971 & 0 & $\mathrm{~F}$ \\
\hline $3289562-5$ & 3478 & 31052 & 908 & $\mathrm{~T}$ \\
\hline $3289562-5$ & $3270 \mathrm{C}$ & 21389 & 1186 & $\mathrm{~T}$ \\
\hline
\end{tabular}

Establish a database on the pneumatic system pressure information that is filled out by the pilot in the pressure data sheet .Because B737NG aircraft pneumatic system uses mechanical control method to control the pressure and temperature, most of the system failure is a gradual process. There is significant amount of time between discovering a problem to function failure. Before the system failure appears, the aircraft would have had signs of something that is wrong for a while.

Table 3. The engine bleed air pressure and corresponding working condition of engine

\begin{tabular}{lccccccc} 
No & \multicolumn{3}{c}{ Left engine } & \multicolumn{3}{c}{ Right engine } & remarks \\
& idle & climbing & cruising & idle & climbing & cruising & \\
\hline B-2693 & 20 & 40 & 42 & 21 & 42 & 45 & \\
B-2694 & 17 & 35 & 38 & 17 & 36 & 37 & \\
B-2695 & 18 & 39 & 37 & 18 & $29 / 35$ & $29 / 35$ & Replace the right \\
B-5020 & 20 & 45 & 45 & 20 & 45 & 45 & \\
B-5147 & 20 & 34 & 38 & 20 & 50 & 42 & \\
B-5739 & 20 & 40 & 40 & 20 & 40 & 40 & \\
B-5741 & 20 & $26 / 45$ & $35 / 45$ & 20 & 42 & 45 & Replace the left \\
B-5747 & 20 & 40 & 40 & 20 & 34 & 40 & PCCV \\
B-5769 & 20 & 40 & 44 & 20 & 43 & 40 & \\
\hline
\end{tabular}

Considering that the period of health check of the pneumatic system is too long, it is not enough to realize the continuous monitoring of the pneumatic system parts, and the consultation sheet of engine bleed air pressure is issued regularly to the pilot the every month. It is regarded as an effective supplementary monitoring method in addition to the test database of critical components. This consultation form is issued regularly to ensure that the engine performance monitor personnel can collect the engine bleed air pressure and the corresponding N1 rotational speed data of the ground idle ,the climbing and cruising stages. The collection of the above data ensures that the engineers can carry out the pressure change of the B737NG aircraft engine bleed air system. Continuous surveillance. When it is found that the bleed air pressure of the engine is beyond the normal range, immediately arrange the workshop workers to carry out preventive health inspection, to replace the affected parts of the pneumatic system in time, and to eliminate the hidden trouble. Thus avoid flight delay caused by 
sudden failure. The engine bleed air pressure and corresponding working condition of engine, as shown in Table 3.

The establishment of the three databases of the pneumatic system helps the continuous monitoring of pneumatic parts performances, and can identify and detect the trend of component failures.

\section{Soft time limit monitoring to the key components of the pneumatic system}

In order to improve the reliability of the pneumatic system and control the maintenance cost effectively, the soft time limit control of the three key components of the pneumatic system (precooler control valve, bleed air regulator, high stage regulator) is carried out.

By referring to the data of the three databases of the pneumatic system, under the normal working condition of the bleed air pressure of the engine, it is not necessary to replace the parts that cannot be tested by a certain function in advance. Based on the collected data of the pneumatic system filled out by the pilot, we can continue to monitor and use the offset components of the pneumatic system health test. It is important to closely monitor the pressure of the affected engine at each stage, take into account the installation time of the component, accurately isolate the faulty parts and replace them at the early stage when a deviation of the bleed air pressure is detected.

The soft time limit control of the key components of the pneumatic system is a dynamic control and management process. It mainly monitors the trend of the pneumatic system data, and intervenes in time before the function failure of the pneumatic system occurs. As a result, the number of occurrence of emergency maintenance and unplanned maintenance and the maintenance cost is reduced.

\section{Conclusions}

1) On the basis of carrying out the health inspection method of the pneumatic system regularly, the reliability of the pneumatic system is improved from two aspects: improving the repair quality of the parts and the monitoring methods of the parts' performance.

2) Monitoring the accessory status and wing time of the pneumatic system can improve the accuracy of the pneumatic system and avoids unnecessary parts replacement. The wing service time of the pneumatic system parts can be greatly improved. According to statistics, the number of failed replacement decreased by $40 \%$. Thus the process not only saved the cost of aviation materials, but also greatly improved the working reliability of the pneumatic system.

3 ) According to the status of system accessories and wing time, the soft time limit is used to change parts to ensure the normal operation of the aircraft.

4) Strengthening quality of component maintenance management to improve the reliability of the aircraft pneumatic system. This conclusion also applies to other systems and other models.

\section{References}

[1] Denfeng She Analysis of the Pneumatic system failure of B737NG engine .Journal of Civil Aviation Flight University of China, 2015 (02): 54-57.

[2] Benxin Zhou Prevention of 737NG Pneumatic system Fault through various measures, Aviation Maintenance \& engineering,2013(5): 53-54.

[3] Boeing. B737-600 / 700 / 800 / 900 aircraft maintenance manual, 15.Oct.2017. 